\title{
Análise da Revisão Cochrane: Reabilitação Cardíaca Baseada no Exercício na Doença Arterial Coronária. Cochrane Database Syst Rev. 2016;1:CD001800
}

\author{
Analysis of the Cochrane Review: Exercise-Based Cardiac \\ Rehabilitation for Coronary Heart Disease. Cochrane \\ Database Syst Rev. 2016;1:CD001800
}

\author{
Rita CARVALHEIRA-DOS-SANTOS*1 ${ }^{*}$ Ricardo-Manuel DELGADO $\otimes^{\star 2,3,4}$, Guilherme FERREIRA-DOS-SANTOS ${ }^{3,4,5}$, \\ António VAZ-CARNEIRO $3,4,6$ \\ Acta Med Port 2019 Jul-Aug;32(7-8):483-487 • https://doi.org/10.20344/amp.11898
}

\begin{abstract}
RESUMO
A doença coronária, responsável por um terço de todas as mortes, é a causa de morte mais comum em todo o mundo. Todavia, com o decréscimo das taxas de mortalidade associadas a esta patologia, observa-se um número crescente de doentes com a doença, com aumento da necessidade de gestão dos seus sintomas e prognóstico. A reabilitação cardíaca, componente essencial do tratamento contemporâneo da doença arterial coronária, é uma intervenção complexa que envolve variadas técnicas, incluindo exercício físico, correção dos fatores de risco cardiovasculares tradicionais, terapia comportamental e apoio psicológico, sendo considerada uma prioridade em países em que a prevalência da doença é elevada. Esta revisão sistemática Cochrane constitui uma atualização de uma revisão Cochrane publicada em 2011, tendo identificado 16 novos ensaios clínicos (3872 doentes), predominantemente em status pós-enfarte agudo do miocárdio e pós-cirurgia de revascularização miocárdica. Incluiu, assim, 63 ensaios clínicos aleatorizados que estudaram a eficácia e o custo-efetividade da reabilitação cardíaca baseada no exercício físico, em pacientes com doença arterial coronária. A confiança na evidência científica variou de baixa a moderada. Dos principais resultados destaca-se o efeito da reabilitação cardíaca, em comparação com a ausência de exercício físico, na redução na mortalidade cardiovascular, todavia sem redução da mortalidade total. Apesar da reabilitação cardíaca ter diminuído o risco global de internamento hospitalar, o mesmo não se verificou para o risco de enfarte, cirurgia de revascularização miocárdica ou intervenção coronária percutânea. Neste artigo sumarizam-se e discutem-se os principais resultados, conclusões e implicações desta revisão sistemática para a prática clínica.

Palavras-chave: Doença Coronária/reabilitação; Ensaios Clínicos Aleatorizados; Exercício; Qualidade de Vida; Reabilitação Cardíaca; Revisão Sistemática
\end{abstract}

\section{ABSTRACT}

Coronary heart disease is the single most common cause of death globally, accounting for one-third of all deaths. However, with falling coronary heart disease mortality rates, an increasing number of people live with the disease and may need support to manage their symptoms and prognosis. A complex intervention that may involve a variety of therapies, cardiac rehabilitation includes exercise, risk factor education, behavioral change, psychological support, and strategies that are aimed at targeting traditional risk factors for cardiovascular disease. Cardiac rehabilitation is an essential part of contemporary heart disease care and is considered a priority in countries with a high prevalence of coronary heart disease. This Cochrane systematic review constitutes an update of a Cochrane review published in 2011, having identified 16 new trials (3872 participants), predominantly post-myocardial infarction and post-revascularization patients. Therefore, it included 63 randomized trials looking at the effectiveness and cost-effectiveness of exercise-based cardiac rehabilitation in patients with coronary heart disease. The quality of the evidence ranged from low to moderate. The main results highlight the effect of cardiac rehabilitation, in comparison with absence of physical exercise, in the reduction in cardiovascular mortality, with no reduction, however, in total mortality. The overall risk of hospital admissions was reduced with cardiac rehabilitation but there was no significant impact on the risk of myocardial infarction, coronary artery bypass graft or percutaneous coronary intervention. This paper aims to summarize and discuss the main results and conclusions of this systematic review, as well as its implications for the daily clinical practice.

Keywords: Cardiac Rehabilitation; Coronary Disease/rehabilitation; Exercise; Quality of Life; Randomized Controlled Trials as Topic; Systematic Review

\section{QUESTÃO CLÍNICA}

Será que a reabilitação cardíaca $(\mathrm{RC})$ baseada no exercício físico, quando comparada com o nível habitual de cuidados de saúde, está associada a diferenças na mor- talidade, morbilidade e qualidade de vida relacionada com a saúde (QVRS) em doentes adultos com doença arterial coronária (DAC)? Adicionalmente, será a RC baseada no exercício físico custo-eficaz?

\footnotetext{
* Co-primeiros autores

1. Serviço de Cardiologia. Hospital Distrital de Vila Franca de Xira. Vila Franca de Xira. Portugal.

2. Serviço de Psiquiatria e Saúde Mental da Infância e da Adolescência. Centro Hospitalar Universitário Lisboa Norte. Lisboa. Portugal.

3. Centro de Estudos de Medicina Baseada na Evidência. Faculdade de Medicina. Universidade de Lisboa. Lisboa. Portugal.

4. Centro Colaborador Português da Rede Cochrane Iberoamericana. Cochrane Portugal. Faculdade de Medicina. Universidade de Lisboa. Lisboa. Portugal.

5. Departamento de Medicina Física e Reabilitação. Centro Hospitalar Universitário de Lisboa Central. Lisboa. Portugal.

6. Instituto de Medicina Preventiva e Saúde Pública. Faculdade de Medicina. Universidade de Lisboa. Lisboa. Portugal.

$\triangle$ Autor correspondente: Ricardo-Manuel Delgado. delgado@campus.ul.pt

Recebido: 06 de fevereiro de 2019 - Aceite: 31 de maio de 2019 | Copyright @ Ordem dos Médicos 2019
} 


\section{OBJETIVOS}

Avaliar a eficácia e a relação custo-eficácia da RC baseada no exercício físico, dicotomizando a análise no caso do exercício físico per se ou em combinação com intervenções psicossociais e/ou de educação do doente, quando em comparação com o nível habitual de cuidados de saúde, na mortalidade, morbilidade e QVRS, em doentes adultos com DAC.

Adicionalmente, averiguar potenciais preditores do nível de eficácia da RC baseada no exercício físico, em pacientes adultos com DAC.

\section{METODOLOGIA}

Esta revisão sistemática (RS) Cochrane é uma atualização de uma RS Cochrane precedente, publicada em 2011, que vem articular a evidência científica pré-existente com a publicada desde então. Com o objectivo de identificar a evidência científica mais recente, os autores desta revisão sistemática refizeram a pesquisa bibliográfica efetuada na anterior RS, através da utilização do Cochrane Central Register of Controlled Trials (CENTRAL) (the Cochrane Library, Issue 6, 2014), com um universo temporal de publicação de dezembro de 2009 a julho de 2014.

Adicionalmente, os autores consultaram ainda as seguintes bases de dados de literatura de ciência médica: MEDLINE (Ovid), EMBASE (Ovid), CINAHL (EBSCO) e Science Citation Index Expanded, utilizando o mesmo universo temporal de publicação (dezembro de 2009 a julho de 2014).

Esta RS Cochrane incluiu ensaios clínicos aleatorizados (ECA) que estudaram o efeito da RC baseada no exercício físico em pacientes adultos com DAC. Os ECAs incluídos para análise apresentaram um período de follow-up mínimo de 6 meses e utilizaram um grupo de controlo, sem prática de exercício físico. A população de participantes incluídos nos ECAs em análise compreendeu indivíduos adultos (> 18 anos) de ambos os géneros, com pelo menos um dos seguintes diagnósticos ou antecedentes cirúrgicos: enfarte agudo do miocárdio (EAM); angina de peito; DAC; cirurgia de revascularização do miocárdio (CRM) e/ou intervenção coronária percutânea (ICP).

Esta RS incluiu os ECAs que reportaram pelo menos uma das seguintes medidas de eficácia (outcomes primários): mortalidade, taxa de EAMs (fatais e não fatais), taxa de revascularização (CRM ou ICP), taxa de internamento hospitalar, QVRS, ou custo.

Dois investigadores efetuaram, de modo independente, um screening inicial de todos os ECAs encontrados durante a pesquisa bibliográfica, com base nos critérios de inclusão e exclusão acima definidos. Um primeiro investigador extraiu informação dos ECAs incluídos e avaliou o seu risco global de viés, enquanto um segundo autor da RS reviu a informação selecionada. A meta-análise de dados foi estratificada em três grupos diferentes, de acordo com a duração do follow-up dos ECAs incluídos para análise: curto-prazo (6 a 12 meses); médio-prazo (de 13 a 36 meses) e longo-prazo (superior a 3 anos).

\section{RESULTADOS}

Esta RS Cochrane incluiu um total de 63 ECAs, correspondentes a um total de 14486 doentes com DAC. Esta atualização identificou 16 novos ECAs, com um total de 3872 novos doentes. A população estudada incluiu maioritariamente doentes em status pós-EAM e pós-CRM, com uma idade média que variou entre os 47,5 e os 71,0 anos. A percentagem de participantes do género feminino foi inferior a $15 \%$ do total de participantes. Globalmente, a qualidade de relato dos ECAs foi baixa, embora com evidência de melhoria nos ECAs mais recentes.

A confiança na evidência científica para as medidas de eficácia reportadas foi avaliada com base nas normas do Grades of Recommendation, Assessment, Development and Evaluation (GRADE) e variou de baixa a moderada, com uma diferença significativa entre as várias medidas de eficácia reportadas pelos diferentes ECAs.

Uma vez que não foram encontradas diferenças significativas no impacto da RC baseada no exercício físico ao longo do período de follow-up, a análise focou-se nos resultados das diferentes medidas de eficácia dos ECAs no seu último momento de avaliação durante o período de follow-up (mediana de 12 meses).

Quando em comparação com o grupo controlo, a RC baseada no exercício físico reduziu a mortalidade cardiovascular em doentes adultos com DAC [27 ECAs, risco relativo (RR) de 0,74, intervalo de confiança (IC) de 95\%: $0,62$ a 0,86$]$. No entanto, a RC baseada no exercício físico não demonstrou reduzir a mortalidade total, quando em comparação com o grupo controlo (47 ECAs, RR de 0,96, IC de 95\%: 0,88 a 1,04).

Quando em comparação com o grupo controlo, a RC baseada no exercício físico reduziu o risco de internamento hospitalar (15 ECAs, RR de 0,82, IC de 95\%: 0,70 a 0,96). No entanto, não se verificou uma diferença significativa no risco de EAM (36 ECAs, RR de 0,90, IC de 95\%: 0,79 a 1,04).

A heterogeneidade estatística da globalidade dos ECAs que reportaram medidas de eficácia de todos os eventos foi baixa, com viés de pequena amostragem apenas para as medidas de eficácia de EAM e taxa de internamento hospitalar.

Dada a heterogeneidade nos métodos de relato utilizados, bem como nas medidas de eficácia propriamente ditas, não foi realizada uma meta-análise de dados para a QVRS. Em cinco dos 20 ECAs que reportaram a QVRS através de medidas de eficácia validadas, os resultados demonstraram uma melhoria significativa para a grande maioria das medidas de eficácia reportadas, no grupo de $\mathrm{RC}$ baseada no exercício físico.

Por último, quatro estudos de avaliação económica baseados em ECAs revelaram que a RC baseada no exercício físico é uma estratégia terapêutica custo-eficaz para a alocação de recursos, em termos de ganho de quality-adjusted life years (QALYs).

Os resultados principais desta RS podem ser consultados na Tabela 1. Por motivos de simplificação, são 
focados os outcomes relativos a um follow-up a mais de três anos, sendo omitidos da mesma os resultados relativos a follow-ups menores. Para uma visualização mais exaustiva dos resultados, aconselha-se a consulta da RS Cochrane original.

\section{CONCLUSÃO}

A presente atualização desta RS vem suportar várias conclusões já apresentadas pela sua versão precedente. Em particular, esta atualização reforça a evidência prévia de que a RC baseada no exercício físico, quando comparada com os protocolos clínicos de $\mathrm{RC}$ sem introdução de exercício físico, reduz o risco de mortalidade cardiovascular - mas não a mortalidade global.

Relativamente a este último ponto, um aspeto novo desta revisão (face à RS Cochrane anterior) merece menção: apesar de se ter verificado uma tendência para a redução na mortalidade global com a RC baseada no exercício (quando comparada a ausência de exercício), essa redução não foi estatisticamente significativa. Dado o caráter sumário do presente Cochrane Corner, optamos por abordar este ponto em maior detalhe apenas nesta secção, avançando com a explicação sugerida pelos autores da RS e partilhada pelos autores do presente artigo. Assim, ocorre que, ao contrário da RS Cochrane de 2011, alguns dos mais recentes ECAs incluídos dizem respeito a populações com DAC mais heterogéneas e, ao terem sido conduzidos num momento em que a terapêutica médica se apresenta otimizada em relação à terapêutica da era dos ECAs incluídos na RS de 2011, se acompanham de uma diminuta oportunidade para se verificarem benefícios incrementais em termos de redução da mortalidade global com a RC baseada no exercício.

Adicionalmente, observou-se que a RC baseada no exercício físico se associou a uma redução significativa da taxa de internamento hospitalar, mas não do risco de EAM ou do recurso a terapêutica de revascularização. Acresce ainda que se verificou, em relação à RS de 2011, o aumento do corpo de evidência que suporta o benefício que a $\mathrm{RC}$ baseada no exercício físico demonstrou no aumento da QVRS.

Relativamente à evidência científica que serviu de base à presente RS, constatou-se que os ECAs mais recentes incluídos para análise apresentaram maior probabilidade de serem bem reportados e de incluírem doentes do género masculino de idade mais avançada, bem como doentes do género feminino. Todavia, destaca-se uma limitação: não obstante esta atualização da RS, a população em análise continua a consistir predominantemente de indivíduos jovens do género masculino, de baixo risco,

Tabela 1 - Reabilitação cardíaca baseada no exercício físico para o tratamento da doença arterial coronária

\begin{tabular}{|c|c|c|c|c|c|c|}
\hline \multicolumn{7}{|c|}{$\begin{array}{l}\text { População: Doentes adultos com doença arterial coronária (DAC) } \\
\text { Intervenção: Reabilitação cardíaca (RC) baseada no exercício físico }\end{array}$} \\
\hline \multirow{2}{*}{$\begin{array}{c}\text { Medidas de eficácia } \\
\text { avaliadas }\end{array}$} & \multirow{2}{*}{$\begin{array}{c}\mathrm{N}^{\circ} \text { de } \\
\text { participantes } \\
\left(\mathrm{N}^{\circ} \text { de estudos) }\right.\end{array}$} & \multicolumn{2}{|c|}{$\begin{array}{c}\text { Número de } \\
\text { eventos / participantes }\end{array}$} & \multirow{2}{*}{$\begin{array}{l}\text { Risco Relativo } \\
\text { (IC 95\%) }\end{array}$} & \multirow{2}{*}{$\begin{array}{l}\text { Teste chi- } \\
\text { quadrado } \\
\text { (Valor-p) }\end{array}$} & \multirow{2}{*}{$\begin{array}{c}\text { Qualidade } \\
\text { da evidência } \\
\text { (GRADE) }\end{array}$} \\
\hline & & Intervenção & Comparador & & & \\
\hline $\begin{array}{l}\text { Mortalidade total } \\
\text { (todos os estudos) }\end{array}$ & $12455(47)$ & $838 / 6424$ & $865 / 6031$ & $\begin{array}{c}\text { RR de } 0,96 \\
(0,88 \text { a } 1,04)\end{array}$ & $0 \%(0,58)$ & Moderada* \\
\hline Follow-up superior a 3 anos & $3828(11)$ & $476 / 1902$ & $493 / 1926$ & $\begin{array}{c}0,91 \\
(0,75,1,10)\end{array}$ & $35 \%(0,12)$ & \\
\hline $\begin{array}{l}\text { Mortalidade cardiovascular } \\
\text { (todos os estudos) }\end{array}$ & $7469(27)$ & $292 / 3850$ & $375 / 3619$ & $\begin{array}{c}\text { RR de } 0,74 \\
(0,64 \text { a } 0,86)\end{array}$ & $0 \%(0,70)$ & Moderada* \\
\hline Follow-up superior a 3 anos & $1392(8)$ & $56 / 690$ & $100 / 702$ & $\begin{array}{c}0,58 \\
(0,43,0,78)\end{array}$ & $0 \%(0,91)$ & \\
\hline $\begin{array}{l}\text { EAM fatais e não fatais } \\
\text { (todos os estudos) }\end{array}$ & $9717(36)$ & $356 / 4951$ & $387 / 4766$ & $\begin{array}{l}\text { RR de } 0,90 \\
(0,79 \text { a } 1,04)\end{array}$ & $0 \%(0,48)$ & Baixa* \\
\hline Follow-up superior a 3 anos & $1560(10)$ & $65 / 776$ & $102 / 784$ & $\begin{array}{c}0,67 \\
(0,50,0,90)\end{array}$ & $0 \%(0,67)$ & \\
\hline $\begin{array}{l}\text { CRM } \\
\text { (todos os estudos) }\end{array}$ & $5891(29)$ & $208 / 3021$ & $212 / 2870$ & $\begin{array}{c}\text { RR de } 0,96 \\
(0,80 \text { a } 1,16)\end{array}$ & $0 \%(0,86)$ & Moderada* \\
\hline Follow-up superior a 3 anos & $675(4)$ & $12 / 333$ & $29 / 342$ & $\begin{array}{c}0,66 \\
(0,34,1,27)\end{array}$ & $18 \%(0,30)$ & \\
\hline $\begin{array}{l}\text { ICP } \\
\text { (todos os estudos) }\end{array}$ & $4012(18)$ & $171 / 2013$ & $197 / 1999$ & $\begin{array}{l}\text { RR de } 0,85 \\
(0,70 \text { a } 1,04)\end{array}$ & $0 \%(0,59)$ & Moderada* \\
\hline Follow-up superior a 3 anos & $567(3)$ & $28 / 281$ & $37 / 286$ & $\begin{array}{c}0,76 \\
(0,48,1,20)\end{array}$ & $0 \%(0,81)$ & \\
\hline $\begin{array}{l}\text { Taxa de internamento } \\
\text { hospitalar } \\
\text { (todos os estudos) }\end{array}$ & $3030(15)$ & $407 / 1556$ & $453 / 1474$ & $\begin{array}{l}\text { RR de } 0,82 \\
(0,70,0,96)\end{array}$ & $34,5 \%(0,10)$ & Baixa* \\
\hline Follow-up superior a 3 anos & $0(0)$ & $0 / 0$ & $0 / 0$ & Não estimado & Não estimada & \\
\hline
\end{tabular}

CRM: cirurgia de revascularização do miocárdio; EAM: enfarte agudo do miocárdio; ICP: intervenção coronária percutânea; RR: risco relativo. 
que sofreram um EAM ou foram submetidos a terapêutica de revascularização.

ECAs ulteriores são necessários para avaliar o impacto da RC baseada no exercício físico em dois grandes espetros de apresentação da DAC: o agudo e o crónico; em relação ao primeiro, elencam-se os doentes coronários de maior risco, e, em relação ao segundo, salientam-se os doentes com angina estável. Como sugestão, estes ECAs deverão incluir medidas de eficácia de QVRS validadas, reportar explicitamente outcomes clínicos primários (tais como mortalidade e taxa de internamento hospitalar) e averiguar os custos e o custo-efetividade dos protocolos clínicos de RC baseada no exercício físico.

\section{COMENTÁRIO DOS AUTORES DA ANÁLISE}

A DAC, a principal causa de morte a nível mundial, é responsável por cerca de um terço de todas as mortes, tendo sido responsável por 7,4 milhões de mortes em 2013, de acordo com dados da Organização Mundial de Saúde.1,2

A RC, uma intervenção complexa que pode envolver uma variedade de estratégias terapêuticas diferentes, incluindo exercício físico, educação sobre e correção dos factores de risco cardiovasculares tradicionais, alterações comportamentais e apoio psicológico, é um componente essencial do tratamento contemporâneo da DAC e deveria constituir uma estratégia de tratamento prioritária em países com elevada prevalência da doença. ${ }^{1}$ Com base em RS previamente publicadas (Clark 20053; Piepoli 20044; Taylor $2004^{5}$ ), a European Society of Cardiology, a American Heart Association e o American College of Cardiology recomendam a RC enquanto estratégia terapêutica de Classe I para o tratamento de doentes que sofreram um evento coronário, com as estratégias baseadas no exercício físico a apresentarem um papel central em todas as recomendações destas instituições. ${ }^{16,7}$

No entanto, apesar do nível de recomendação internacional da RC no geral, e das estratégias baseadas no exercício físico em particular, enquanto componentes fundamentais do tratamento de pacientes com DAC (em particular após EAM, terapêutica de revascularização coronária, ou em doentes com angina pectoris), a literatura internacional demonstra que uma percentagem significativa destes pacientes ainda não recebe este nível de cuidados. ${ }^{8} \mathrm{Em}$ Portugal, somente $8 \%$ dos doentes que recebem alta do hospital após EAM recebem integração em programas de RC. Este aspeto contrasta grandemente com a média Europeia de $30 \%$, ou mesmo com a média dos EUA de $20 \%-30 \% .{ }^{9}$ Ora, além de estarmos perante uma janela de oportunidade para a melhoria do acesso de maneira equitativa a este tipo de intervenção, em que se pretende não apenas o aumento do número de programas a nível nacional mas, igualmente, a elevação da sua qualidade média e monitorização, existem fatores que têm limitado o acesso a estes programas: nomeadamente culturais, organizacionais e financeiros. De destaque, na última análise efetuada pela Sociedade Portuguesa de Cardiologia, em 2013 - 2014, existiam 23 centros de RC em território nacional, todavia maioritariamente distribuídos na Grande Lisboa e Grande Porto. ${ }^{9}$ Apesar da realidade portuguesa ser particularmente sensível, a questão da referenciação a programas de RC continua a ser um desafio numa significativa proporção dos países desenvolvidos. Neste sentido, e enquanto se aguarda a extensão da capacidade dos programas de $\mathrm{RC}$ já existentes, pode considerar-se a ponderação de programas de RC home-based para doentes de baixo risco e a exploração de meios tecnológicos atuais com a tónica subjacente na referenciação sistemática de todos os doentes com DAC, quer após evento relacionado motivador de internamento quer em contexto ambulatorial. ${ }^{10}$

Metodologicamente, e apesar de a RS em análise oferecer um elevado grau de qualidade metodológico, a condução de uma RS não é isenta de limitações. Em particular, a consideração da inclusão de ECAs envolvendo populações nem sempre homogéneas, a par da reportagem nem sempre standardizada dos outcomes em questão repercute-se por vezes na impossibilidade de comparação estatisticamente correta de algumas questões que, intuitivamente, o raciocínio clínico poderia julgar já conhecer. Todavia, é aqui que reside o poder e a ciência de uma abordagem da evidência através de uma RS: quando a evidência encontrada cumpre determinados preceitos que permitam a comparação, essa é feita com robustez e permitindo conclusões que, na hierarquia da evidência médica, se assumem de valor superior relativamente ao de um case report decorrente do registo da observação clínica quotidiana. Para além das limitações metodológicas explanadas, os novos ECAs incluídos ocorreram maioritariamente numa era em que a otimização da terapêutica médica se repercute em benefícios significativos ao nível de vários dos outcomes analisados. Isto diminui a margem para benefício incremental do exercício físico, o que faz com que seja compreensível que a revisão em análise não tenha chegado a uma conclusão definitiva quanto, por exemplo, ao nível (por vezes intitulado de dose) de exercício físico, ou ao período ideal de prescrição de exercício físico para o qual um trade-off custo-benefício se encontre otimizado. Essas são questões relevantes a ser tidas em conta em futuras RS, bem como no desenho de ECAs a ocorrer posteriormente.

Finalmente, há que considerar o facto de a RC baseada no exercício físico ser uma estratégia terapêutica extremamente segura. Em 2006, Pavy e colaboradores publicaram um estudo observacional, com um total de mais de 25000 participantes com DAC em tratamento segundo um protocolo clínico de RC baseada no exercício físico, no qual reportaram uma taxa de ocorrência de um evento adverso cardíaco por cada 50000 horas de exercício físico, o equivalente à ocorrência de uma paragem cardíaca por milhão de horas de exercício físico por doente. ${ }^{11}$ Não obstante a segurança dos protocolos clínicos atuais de RC baseada no exercício físico, a British Association for Cardiovascular Prevention and Rehabilitation adverte para uma avaliação cuidadosa de pacientes com angina instável, disritmias ventriculares não controladas e insuficiência cardíaca grave (Classe IV/IV da New York Heart Association) a serem 
considerados para um protocolo de RC baseada no exercício físico. ${ }^{1,12}$

\section{IMPLICAÇÕES PARA A PRÁTICA CLÍNICA}

1. Esta RS vem demonstrar que, apesar de não diminuírem a mortalidade global, os protocolos clínicos de RC baseada no exercício físico diminuem a mortalidade cardiovascular e a taxa de internamento hospitalar (adicionalmente, também reduzem os custos económicos associados a estes eventos), além de produzirem ganhos na QVRS de doentes com DAC. Estes benefícios ficaram demonstrados no subgrupo de doentes jovens do género masculino, que sofreram um EAM ou foram submetidos a terapêutica de revascularização.

2. Apesar de os ECAs mais recentes incluírem uma maior proporção de doentes do género feminino e doentes do género masculino de idade mais avançada, a extrapolação da evidência científica aqui demonstrada a doentes de grupos sub-representados na população em estudo nesta

\section{REFERÊNCIAS}

1. Anderson L, Thompson DR, Oldridge N, Zwisler AD, Rees K, Martin N, et al. Exercise-based cardiac rehabilitation for coronary heart disease. Cochrane Database Syst Rev. 2016;1:CD001800.

2. World Health Organization. The top 10 causes of death. Fact sheet No. 310. Updated May 2014. Geneva: WHO; 2014.

3. Clark AM, Hartling L, Vandermeer B, McAlister FA. Metaanalysis: secondary prevention programs for patients with coronary artery disease. Ann Intern Med. 2005;143:659-72.

4. Piepoli MF, Davos C, Francis DP, Coats AJ, ExTraMATCH Collaborative Exercise training meta-analysis of trials in patients with chronic heart failure (ExTraMATCH). BMJ. 2004;328:189.

5. Taylor RS, Brown A, Ebrahim S, Jolliffe J, Noorani H, Rees K, et al. Exercise-based rehabilitation for patients with coronary heart disease: systematic review and metaanalysis of randomized controlled trials. Am J Med. 2004;116:682-92.

6. Balady GJ, Ades PA, Bittner VA, Franklin BA, Gordon NF, Thomas $\mathrm{RJ}$, et al. Referral, enrollment, and delivery of cardiac rehabilitation/ secondary prevention programs at clinical centers and beyond: a presidential advisory from the American Heart Association. Circulation. 2011;124:2951-60.

7. Perk J, De Backer G, Gohlke H, Graham I, Reiner Z, Verschuren M, et al. European Guidelines on cardiovascular disease prevention in clinical
RS deve ser feita cautelosamente, em particular em pacientes com angina pectoris, DAC de alto risco (angina instável) e doentes com comorbilidades major. A correta adequação de um protocolo clínico de RC baseada no exercício físico a estes grupos de doentes permanece ainda uma questão de julgamento clínico.

3. A evidência científica atualmente disponível é parca no que concerne à comparação direta de protocolos clínicos de RC baseada no exercício físico com protocolos clínicos de RC que combinam estratégias de exercício físico juntamente com intervenções psicossociais e/ou de educação dos doentes. ${ }^{1} \mathrm{Na}$ ausência de dados concretos que permitam fazer uma comparação direta entre o custo-eficácia de protocolos clínicos de RC baseada no exercício físico com protocolos clínicos de RC baseada em abordagens psicossociais e de educação do doente, é racional adequar a prática clínica diária tendo em atenção o custo económico de cada uma das intervenções disponíveis ao clínico.

practice (version 2012). The Fifth Joint Task Force of the European Society of Cardiology and Other Societies on Cardiovascular Disease Prevention in Clinical Practice (constituted by representatives of nine societies and by invited experts). Eur Heart J. 2012;33:1635-701.

8. Bethell H, Lewin R, Evans J, Turner S, Allender S, Petersen S. Outpatient cardiac rehabilitation attendance in England: variability by region and clinical characteristics. J Cardiopulm Rehabil Prev. 2008;28:386-91.

9. Abreu A, Mendes M, Dores H, Silveira C, Fontes P, Teixeira M, et al. Mandatory criteria for cardiac rehabilitation programs: 2018 guidelines from the Portuguese Society of Cardiology. Rev Port Cardiol. 2018;37:363-73.

10. Pastormelo LE, Aimo A, Piepoli M, Emdin M. The bottleneck of cardiac rehabilitation for patients with coronary artery disease: How to overcome. Eur J Prev Cardiol. 2018;25:1239-41.

11. Pavy B, Iliou MC, Meurin P Tabet JY, Corone S; Functional Evaluation and Cardiac Rehabilitation Working Group of the French Society of Cardiology. Safety of exercise training for cardiac patients: results of the French registry of complications during cardiac rehabilitation. Arch Intern Med. 2006;166:2329-34.

12. British Association for Cardiovascular Prevention and Rehabilitation. The BACPR standards and core components for cardiovascular disease prevention and rehabilitation, $2^{\text {nd }}$ ed. London: BACPR; 2012.

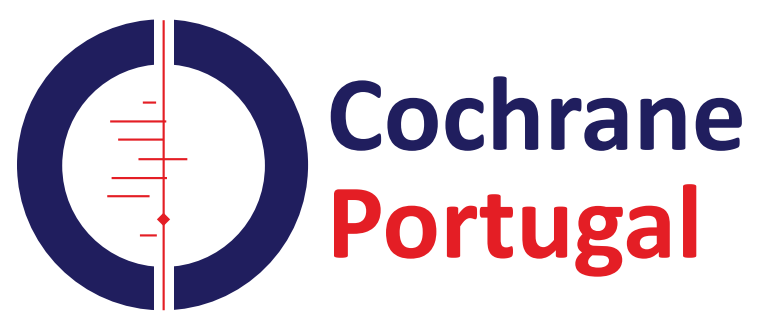

